\title{
LAS PRUEBAS DE LA VEJEZ EN MUJERES. EL CASO DE SANTIAGO DE CHILE Y MONTEVIDEO ${ }^{1}$
}

\section{THE TESTS OF OLD AGE IN WOMEN. THE CASE OF WOMEN FROM} SANTIAGO DE CHILE AND MONTEVIDEO

\section{Regina Praxedes Obreque Morales²}

Resumen

\begin{abstract}
El artículo profundiza en los modos de individuación de las mujeres que vivencian o experimentan la vejez en Santiago de Chile y Montevideo, a la luz de las pruebas y desafíos que la actual generación se encuentra afrontando en esta etapa de la vida. Con un resultado de once pruebas, las que a través de dinámicas culturales, epocales e institucionales, evidencian hallazgos sobre procesos de individuación que se encontrarían experimentando las mujeres en estas dos ciudades latinoamericanas, caracterizadas en las últimas décadas por contar con una población envejecida.
\end{abstract}

Palabras claves: Generación; Individuación; Mujeres; Pruebas; Vejez.

Abstract

\begin{abstract}
The article delves into the ways of individuation of the women who experience old age in Santiago de Chile and Montevideo, in light of the trials and challenges that the current generation is facing at this stage of life. With a result of eleven tests, which through cultural, epochal and Institutional dynamics, evidence finds about processes of individuation that women in these two Latin American cities, characterized in the last decades, would experience experiencing, due to having aging population.
\end{abstract}

Key words: Generation; Individuation; Women; Tests; Old age.

\footnotetext{
${ }^{1}$ El artículo muestra los principales resultados del estudio Pruebas en la vejez. Análisis del proceso de individuación en mujeres que vivencian la vejez. El caso de Santiago de Chile y Montevideo. Documento de trabajo de tesis doctoral en Estudios americanos USACH/IDEA.

${ }^{2}$ Socióloga. Universidad de Santiago de Chile. E-mail: regina.obrequem@usach.cl
} 


\section{Introducción}

Al analizar la relación entre vejez eindividuación, en específico en la experiencia llevada a cabo por mujeres que habitan en dos ciudades sudamericanas que se han caracterizado por el acelerado envejecimiento de su población: Santiago de Chile y Montevideo (CEPAL/ECLAC, 2014), es posible evidenciar los modos sociales en que las grandes transformaciones societales que configuran el proceso de la vejez, se inscriben en las experiencias de vida individual de las mujeres.

Proceso acompañado de transformaciones demográficas, que conllevaron a que dicho proceso se identifique con un aumento de la esperanza de vida prácticamente en todos los países latinoamericanos; con un incremento de sobrevida que en la actualidad permite la coexistencia de tres y hasta cuatro generaciones familiares en buenas condiciones físicas y mentales, planteando nuevos desafíos individuales y societales, convocados a escenarios de convivencia y de relaciones intergeneracionales (ONU, 2012; RLG, 2009).

Lo anterior fue abordado por medio del relato de mujeres mayores de ambas ciudades ${ }^{3}$, persiguiendo el objetivo de analizar y comparar las experiencias de dichas mujeres sobre las pruebas de la individuación dadas en su vejez, soportes y estrategias. Indagando en las pruebas y la relación con los procesos de transformación socio/individual. Relatos vistos a la luz de la constitución del individuo mujer en la vejez y los desafíos que aquello conlleva, desde una noción de prueba, que entiende de retos que las sociedades imponen a los individuos y por los cuales son empujados a procesos de individuación (Martuccelli, 2007), desarrollando soportes y estrategias para enfrentar dichas pruebas. La prueba será, entonces, una categoría analítica que permite establecer un vínculo entre las grandes transformaciones estructurales y las experiencias subjetivas de los individuos (Araujo y Martuccelli, 2012).

En este sentido, diversos estudios sobre procesos de individuación resultan un aporte sustancial en esta reflexión, en especial los trabajos de Martuccelli (2007, 2010), Martuccelli y Singly (2012), Vincent Caradec en Francia (2004). En Chile, el trabajo desarrollado por Kathya Araujo y Danilo Martuccelli sobre las nueve pruebas de los chilenos el año 2012.

Lo anterior nos sitúa en la discusión y el debate gerontológico sobre la carencia de producción de recursos y modelos teóricos que abordaron la vejez, como proceso individual biologicísta, propiciando enfoques empiristas e intervencionistas (Yuny y Urbano, 2008). Enfoques que aludían a sociedades impregnadas por el viejismo

\footnotetext{
${ }^{3}$ Estudio de caso, en el cual se entrevistó un total de 32 mujeres -entrevista semi estructurada- tomando como criterio muestral la selección de dos tramos etarios, el tramo de 60 a 80 años de edad, quienes conformarían el grupo "entrando en la vejez", y el tramo de personas de 80 y más años, conformando el grupo "en edades avanzadas". Para ambos grupos se consideró además, la pertenencia a sectores socioeconómicos bajos y/o medios; la actividad económica pasiva de dueña de casa (ama de casa) y/o jubilada.
} 
(Barros, 1979; Butler, 1969), que refuerzan valores que confrontan juvenismo y viejismo, confrontando dinámicas intergeneracionales en sociedades envejecidas de la región (RLG, 2009). Enfoques que, además, enfrentaban más bien a la mirada de las vejeces (Lalive d'Epinay, 1983) y la noción que la integra como el resultado de la estructura de desigualdad existente en la sociedad (Bury, 1996).

En este escenario, la individuación de las adultas mayores se ve entrelazada con desafíos o pruebas que conllevan la constitución de individuos a lo largo de la vida. Acompañado además, de cambios estructurales neoliberales que colocan en tensión el acceso a recursos sociales y materiales, lo cual se expresa en pruebas que deben enfrentar las mujeres de esta generación.

Proceso que, en la última década, desarrolla un despliegue de marcos jurídicos internacionales y nacionales en derechos de protección de las personas catalogadas como adultas mayores y que es caracterizado por su marcada feminización (Pérez Díaz, 2000). Lo anterior plantea desafíos particulares para las mujeres mayores, considerando que aquellas viven las consecuencias más profundas que acompañan el cambio sociodemográfico en la región (CELADE/ CEPAL, 2011).

El desarrollo institucional para hacer frente a los desafíos del envejecimiento demográfico muestra que Chile y Uruguay poseen un tratamiento diferenciado de políticas públicas en torno a la vejez (Huenchuan, 2012). Chile ha logrado un desarrollo reciente en la materia, resultado de un proceso construido sobre la base de una nueva relación sistema público/estado de bienestar, reproduciendo por décadas el concepto de anciano reducido a la función familiar, consanguínea y doméstica del mundo privado. Siendo, en las últimas décadas, la propia denotación de adulto mayor lo que viene a representar una voluntad de cambio cualitativo en el sistema de relaciones sociales y políticas, construidas a partir de las características propiamente etarias del sector. Debido a este cambio de concepto, se crea, a través de la Ley 19.828 del año 2002, el Servicio Nacional del Adulto Mayor, SENAMA.

Uruguay exhibe un desarrollo institucional que se enmarca en un proceso de reforma social centrado en el ámbito de los derechos ciudadanos, destacando en la actualidad competencias en su rol fiscalizador sobre establecimientos de cuidados. El país cuenta con INMAYORES (Instituto Nacional de las Personas Mayores), órgano coordinador, desde el año 2005, de políticas públicas hacia la vejez. Una función que era realizada con anterioridad directamente por el Ministerio de Desarrollo Social (MIDES, 2013). La institución posee una visión que amplía su paradigma sobre la base del diagnóstico de una lucha sindical de los jubilados en materia de seguridad social. 


\section{De la feminización de la vejez al estudio generacional de las mujeres}

Algunos autores han planteado el proceso de feminización de la vejez como inversión de roles en la sociedad moderna (Pérez Díaz, 2000), con nuevas subjetividades de género, comprometiendo proyectos personales e individuales (Debert, 1994; Pérez Ortiz, 2003). Dicho fenómeno, producto de los procesos sociodemográficos que caracterizan a las sociedades contemporáneas, nos sitúan en nuevas subjetividades de género en esta etapa de la vida. Subjetividades y cambios de roles tradicionales que transitan hacia la cultura moderna, y que lentamente se ve feminizada.

Se trata, entonces, de un fenómeno demográfico espontáneo, que da cuenta de una mayor proporción de mujeres en la vejez. Fenómeno que comienza a generar grietas en la dinámica social, a través de la inversión de roles en esta etapa de la vida (Pérez Díaz, 2000). Aquello muestra soportes para la sociedad en su conjunto, socializando la experticia del manejo doméstico y privado, en particular el aprendizaje del cuidado, un dominio por excelencia de dicha generación de mujeres. Lo sensible del mundo privado se pone en escena hegemónica, las mujeres en la última etapa de la vida visibilizan su contribución social.

Más allá de las diferencias y enfoques, lo relevante del recorrido teórico del estudio gerontológico es el tratado de la vejez como problemática social, enfrentándose al mecanicismo positivista que alumbraba su lectura. Considerando en ello, ser una construcción social que avanza sobre teorías predominantes instaladas en la literatura gerontológica, desde enfoques biomédicos hacia miradas bio-psico-sociales (Fernández-Ballesteros, 2009; Herrero, 2001), comenzando a incorporar la dimensión cultural, social y política en el estudio del envejecimiento y la vejez (Ferrer, 2005).

Históricamente, los nuevos escenarios teóricos dan cuenta, por un lado, de la crisis del paradigma científico dominante y, por otro, de la aparición de las ciencias sociales y culturales. Lo que dio paso a la producción de nuevos enfoques en los modos de construcción teórica del proceso de envejecimiento, dejando atrás el exacerbado empirismo e intervención social que impidió que investigaciones gerontológicas generaran recursos, deteniéndose en la lectura individual biologícista, instalando una visión decremental y deficitaria de la vejez (Yuni y Urbano, 2008).

Desde este escenario, se comprende la necesidad que sociedades envejecidas se conviertan en sociedades más humanas (Neugarten, 1999), logrando volcarse a mirar realidades heterogéneas, abordadas desde enfoques interdisciplinarios (biomédico, comportamental, social, espiritual), desde subcampos que intercambian esferas societales (geopolítica, género, generaciones, étnica), 
cobrando sentido la demanda gerontológica de la vida interior de las personas mayores en sus significados y experiencias (Achenbaum, 1997), en el proyecto biológico del organismo y el proyecto individual de la conciencia (Baltes, 1997), abriendo paso a un campo de acción teórico eidético de la vejez en el estudio de las vidas.

Autores concuerdan que la perspectiva del ciclo de vida permitió observar el envejecimiento en relación a la biografía personal y el contexto histórico (Elder y Johnson, 2002; Lalive D’Epinay, Cavalli, y Guilley, 2005; Quadagno, 1999). Como indicaría Neugarten (1999), un todo constituyente que avanza desde el origen hasta el fin de la vida "en los procesos de cambio desde la infancia hasta la vejez" (p. 380).

En este espacio, Caradec (2004) propone caracterizar la vejez con un test que marque la tensión entre la separación del mundo y mantenerse en el mundo, conservarse actualizado en torno a las áreas de conocimiento del mundo. Se plantea la prueba de la vejez, desglosada en tres dimensiones principales: una relación global pragmática (e interpretativa de lo social), la relación con uno mismo y el sentido de pertenencia en el mundo (Caradec, 2004). ${ }^{4}$

Diversos autores -al finalizar la década de 1980- comienzan a analizar la conexión entre edad y relaciones de género (Arber y Ginn, 1990; Levy, 1988; Reinharz, 1989). Según McMullin (1996), un hecho que sorprende en los enfoques de género es que el predominio de mujeres en la vejez no haya sido material de análisis.

A partir de entonces, se inicia el recorrido en la producción de investigaciones sobre las mujeres mayores con diferentes enfoques metodológicos (McMullin, 1996; Peace, Holland, y Kellaher, 2006). Las sociólogas feministas, inclinadas a métodos cualitativos, serán quienes contextualizan la experiencia de las mujeres y se preocupan por conseguir cambios en este ámbito (McMullin, 1996). A lo que Bury (1996) agrega que dichas demarcaciones son el resultado procesual de la división del trabajo y la estructura de desigualdad existente en toda sociedad y en todo momento.

Finalmente, desde contextos hispanoamericanos, diversas autoras desarrollan estudios sobre vejez, género y generaciones (Freixas, 2007; Freixas, Luque, y Reina, 2009; Maqueira, 2002; Ortega, 2002; Osorio, 2010; Pérez Ortiz, 2006; Rodríguez, 2002). Al respecto, Osorio (2010) sostiene que las categorías sociales

\footnotetext{
${ }^{4}$ Desde el enfoque de las pruebas, Vincent Caradec plantea tres estrategias analíticas que la sociología de la vejez ha desarrollado. Una sociología que observa el fenómeno como categoría social contribuyendo a la representación social de la vejez a través de estrategias analíticas. Una construcción social de la vejez que revela cómo la sociedad piensa, organiza y da forma a esta edad de la vida. Y la estrategia estadística, que identifica y describe la diversidad interna del grupo de edad, segmentándola-jóvenes jubilados o personas mayores que viven en instituciones. Véase Vincent Caradec, 2004.
} 
de la vejez logran históricamente su organización en base a la diferenciación de clase, género y edad.

En un escenario que plantea necesidades contemporáneas de las mujeres mayores, sujetas a modelos de vida en torno a un sistema de género diferencial entre mundo privado (reproductivo) y mundo público (productivo). Lo que nos habla de una nueva generación de mujeres, que extiende opciones vitales desde el campo emotivo, sacrificándose el proyecto individual ante lo social y colectivo (Rodríguez, 2002).

De esta manera, el enfoque del ciclo de vida ha permitido clasificar a los individuos (recursos-poder), siendo la edad y el género variables esenciales para comprender la realidad de las mujeres mayores (Maqueira, 2002).

Lo anterior plantea retos en una sociedad androcéntrica, en torno a la vida de las mujeres mayores, su salud y bienestar, en el entendido que los prejuicios que sostienen su imagen social se vuelven un obstáculo para una población que va en aumento, en mejores condiciones físicas y mentales de las generaciones que le preceden (Freixas, Luque, y Reina, 2009).

La lectura del ciclo vital en el análisis de la vejez ha sido uno de los grandes aportes a la discusión gerontológica de las últimas décadas, en la que la cuestión de género constituye una interrogante mayor desde los diferenciales que tienen las etapas de la vida, desde la óptica masculinizada predominante en las miradas de los estudios psicológicos. La vejez constituye así, para las autoras, un proceso que desentraña las desventajas de las mujeres en relación a los varones, situando el discurso feminista como adquisición de capitales (culturales, sociales y económicos) para dicha generación, lo que les otorga una ventaja social (Freixas, Luque, y Reina, 2009).

Asimismo, generación y procesos feministas van a la par con su crecimiento y visibilización, dándose en el derecho a voto, a diferencia de los países modernos, como un valor significativo para la población latinoamericana, lo que permitió la igualación en la carrera por obtención de derechos de las mujeres en relación a todos los grupos sociales, quienes rápidamente lograron la inserción en el mundo laboral y las creaciones de legislaciones a su favor (Pérez Ortiz, 2006). 


\section{Resultados}

Los principales resultados del estudio identificaron once pruebas de las mujeres en la vejez, distribuidas en dos dimensiones o niveles: Cultural epocal y Político institucional. Respecto a la primera dimensión, se registraron: la prueba de la experiencia del modelo de vejez; la prueba familiar; la prueba de la transformación y el cambio; la prueba de la vida moderna; la prueba de la experiencia de la vida sexual; la prueba de la fe en la vida moderna.

Mientras que en la dimensión política institucional se identificaron: la prueba de la integración social; la prueba de la "economía de guerra"; la prueba de los cuidados; la prueba de la atención de salud y la prueba del uso de la ciudad.

Situándonos en la dimensión cultural epocal, la prueba del modelo de vejez se presenta con el soporte que busca diferenciarse del modelo de vejez que habían construido los padres de las actuales mujeres mayores. Las mujeres en edades avanzadas explican que, en la distancia epocal del modelo de vejez de sus padres, se presenta el soporte de la familia moderna nuclear y las transformaciones epocales, vistas en su estética, recordado en la moda, desde el atuendo, pasando por las conductas, hasta llegar a las estructuras del cuidado. En un contexto que describe familias y hogares desde modelos extensivos a familias monoparentales $y$, con ello, sus valores culturales sobre el cuidado hacia los mayores.

Las mujeres entrando en la vejez, en el manejo tecnológico de los sistemas modernos, han identificado el soporte para enfrentar la prueba del modelo de vejez. Lo que va desde una vejez pasiva a una vejez activa. Las santiaguinas evidenciaron en el alto costo de la vida moderna y los valores de intercambio que se juegan en ella, los avances en los procesos de modernidad en que los contextos periféricos interactúan en el espacio de la vida cotidiana, colonizándolo. También una actitud pragmática de la vida permitió el mejor manejo de los sistemas modernos burocráticos, lo que se entendió como estrategias que juegan de manera favorable al momento de enfrentar la prueba del modelo de vejez.

En relación a la prueba de la familia, las mujeres entrando en la vejez han recurrido a la transmisión de valores intergeneracionales transmitidos a los jóvenes como los soportes que contribuyeron a enfrentar dicha prueba. Se trata de estrategias éticas y de conductas morales que toman como umbral de dignidad, la autonomía en el centro del mecanismo al enfrentar la prueba familiar: "yo me las arreglo con lo mío". Por medio de una autonomía, principalmente económica, en el acceso a espacios públicos que generan dinámicas de realización personal, que en los sectores medios significa: compartir, salidas y amistades; y en los sectores populares: encuentros familiares. 
Se consigna una etapa de adaptación que acompaña la prueba familiar para las mujeres en edades avanzadas. El tiempo y la resignación permiten conformar los mecanismos para constituir la estrategia que enfrenta la prueba de la familia en la vejez de las mujeres de Santiago y Montevideo.

En cuanto a la prueba de la transformación y el cambio en la vejez, esta presenta un soporte que asimila del mensaje del envejecimiento activo en la sociedad moderna, las prácticas de ejercicios del cuerpo, revelando estrategias para hacer frente a dicha prueba. Las mujeres entrando en la vejez constatan en el uso de terapias alternativas de salud un soporte sustancial al enfrentar la prueba. Las mujeres en edades avanzadas nos hablan de un soporte en referencia al nido vacío. En particular, un soporte que remite a la autonomía emotiva de las mujeres, que ante las grandes transformaciones individuales que contrae la etapa de la vejez (separación, la soledad y conflictos familiares y económicos), toman de ella el valor de la libertad y el uso del tiempo libre como estrategias centrales para enfrentar la prueba de la transformación y cambio en su vejez.

La prueba de la vida moderna, que es caracterizada por el desarrollo tecnológico, generó soportes vinculados a la relación intergeneracional. Las mujeres entrando en la vejez identifican el soporte en el uso de las tecnologías, logrando el manejo de los tiempos y la dinámica que trajo consigo la vida moderna. Acompañado de una relación de pérdida y ganancia que deja el impacto de la tecnología en sus vidas. Ganancias, especialmente respecto al conocimiento, sobre técnicas y uso del lenguaje tecnológico aportadas principalmente por las generaciones más jóvenes, que logran ser comunicados y adquiridos por las mujeres mayores, a través de relaciones intergeneracionales.

Por su parte, las mujeres en edades avanzadas de ambas ciudades, en particular las jubiladas de sectores medios, consignan en el desarrollo tecnológico un hacer nuevo de la vejez, donde la prueba de la vida moderna desarrolla sus propios soportes en el modernismo y la modernización de los procesos tecnológicos como efectos globalizantes (Castells, 2005), lo cual incide en el diario vivir. Lo señalado hace referencia a estrategias aludidas por las mayores, que por medio de viajes, cruceros y encuentros con otras culturas, logran conceptuarse en el aporte tecnológico de la vida moderna.

Respecto de la prueba de la experiencia de la vida sexual de las mujeres en la vejez, se revela en las mayores de edades avanzadas, en particular de sectores medios, la distancia de estigmas en torno a la sexualidad, con una serie de recursos para enfrentar la prueba, dejándolo a una actitud abierta y moderna. Las mujeres de sectores medios enfrentan la prueba de la sexualidad con recursos como consoladores, los cuales adhieren a diversas estrategias que ofrece la tecnología en la vida actual. Mientras que para las mujeres entrando en la vejez, el desarrollo 
de soportes médicos ante la prueba de la sexualidad se impone, en particular para las casadas, normalizando la conducta a través del mandato médico.

Por su lado, la prueba de la experiencia de la fe muestra en mujeres de edades avanzadas, en su mayoría católica, un soporte dirigido a una nueva práctica etérea personal de directa comunicación con lo divino. Mientras que las mujeres entrando a la vejez, en particular montevideanas, manifiestan en su condición, por naturaleza agnóstica, el soporte para enfrentar la prueba. Se consignó que la fe católica o en algunos casos evangélicas, es la más referida por las mujeres en edades avanzadas, a través del clásico rito de la misa.

En Uruguay existe una clara inclinación de las mujeres hacia la no práctica religiosa, el ateísmo y una valoración negativa hacia las religiones actuales (principalmente la católica). Con soportes que van desde la nula angustia existencial para las agnósticas, pasando por prácticas hibridas de carácter teosófico (entre rezos, santos y taichí) para las mujeres entrando en la vejez. Son las formas que adquieren los soportes de la prueba de la fe en las mujeres mayores de ambas ciudades.

Respecto a la dimensión institucional, esta presenta en la prueba de la integración social, el desarrollo de soportes psicosociales, tales como identidad, realización personal, autoestima. Desde el enfoque de una política de vida (Giddens, 1994), se daría paso a estrategias de participación social, alfabetización, o nuevas perspectivas, lo que se presenta como objetivos de superación en los relatos de las mayores.

Se consignó, así, que los diversos mecanismos que logran integrar a las mujeres al espacio social moderno y público, hacen alusión a ofertas de actividades vinculadas a la vida cultural y recreativa. Por su lado, las mujeres en edades avanzadas, desarrollaron soportes basados en la valoración de sobrevida, es decir, en el desarrollo de nuevas perspectivas a través de objetivos de superación, los cuales se presentan como estrategias para enfrentar la prueba de la integración social. Mientras que las mujeres entrando en la vejez identifican en la participación social, el soporte para enfrentar dicha prueba (en particular, las dueñas de casa), ven en ello una proyección natural dada desde la vida comunitaria (iglesia, juntas de vecinos, clubes).

En este escenario, la dimensión política institucional, nos trae la prueba de la "economía de guerra", con soportes previsores que hacen alusión a estrategias referidas al control y manejo de la economía del reajuste doméstico. Un soporte transversal en ambas etapas de la vejez. En otros términos, soporte preventorio para enfrentar esta prueba. Aquello se ha acompañado del trabajo de baja calificación al cual se adscriben algunas mujeres en esta etapa de sus vidas. El nuevo presupuesto representó un cambio radical, lo que es parte sustancial 
de la prueba. De manera transversal, gracias a estrategias previsoras que ellas construyen, logran hacer frente a la prueba de la "economía de guerra".

Respecto a la prueba del cuidado en la vejez, se disputó el soporte de la autonomía con las anteriores pruebas, con un soporte legítimo que enfrenta la prueba de los cuidados en la vejez de las mayores, lo que amenaza directamente la posibilidad de ser actor capaz de autosostenerse (Martuccelli, 2007).

Las mujeres entrando en la vejez, identifican soportes diferenciales al momento de enfrentar la prueba del cuidado en ambas ciudades. La familia en las santiaguinas, y la institucionalidad del asilo en las montevideanas. Así como vivir con los hijos o en el asilo, son las proyecciones que representan en sus relatos respecto a sus futuros cuidados.

Se consigna que la racionalidad que asimiló la institucionalidad del asilo en las montevideanas,se propaga lentamente en el pensar de las santiaguinas que se encuentran entrando a la vejez, como soporte alternativo ante la nueva dinámica familiar y su cambio estructural nuclear con tendencia monoparental. Por su lado, el relato de las mayores en edades avanzadas reveló que estas acuden a las redes que han construido en sus historias de vidas, dejando en las manos de los familiares que se acate su decisión del cuidado.

Por otro lado, la prueba de la atención de salud muestra cómo ambas ciudades evidencian un desarrollo institucional en la materia, distanciándose del enfoque biomédico, entregando servicios sanitarios que dan cuenta de mecanismos modernos de derechos de las personas mayores (Huenchuan, 2012). Sin embargo, los soportes que consignó el estudio para enfrentar dicha prueba muestran la fragilidad real del sistema de salud y la mala calidad de su servicio para las mayores. Lo anterior da cuenta de una discriminación territorial y económica en su acceso, particularmente en Santiago. Diferencia de acceso, según el sector socioeconómico, como también el tipo de pensiones, mostrando las deficiencias del sistema de salud, en miras de una sociedad que propicie las condiciones de salud y bienestar para vivir la vejez (ONU, 2002).

Las mujeres en edad avanzada, en particular en Montevideo, manifiestan una valoración positiva del sistema de salud público, al beneficiarse de la atención médica a domicilio. Lo anterior ha llevado a mujeres santiaguinas en edades avanzadas a valorar positivamente este contexto, permitiéndoles enfrentar la prueba de la salud solo con algunas condicionantes propias como son los exámenes y otras complejidades médicas que quedan en suspenso, definidas mejor o peor según el grupo social en el que se ubique.

Las mujeres entrando en la vejez consignan en la búsqueda de alternativas de salud un soporte para enfrentar la prueba. De esta manera, se recurre a 
métodos alternativos dependiendo de los sistemas de creencias, que a su vez se derivan de fuertes experiencias de desamparo institucional, de accidentes, que lleva a las mujeres hacia métodos médicos diferentes al tradicional, en base a un cuestionamiento crítico de la falta de una salud integral, en particular los geriatras. ${ }^{5}$ Se constata, en Santiago, que las pensionadas de carabineros o de las mujeres que viven en las comunas más acomodadas de la ciudad, la atención de salud se adquiere en hospitales de alta complejidad. También se dispone de la salud privada, en clínicas, o en centros integrales de salud, pero con enfoques alternativos a éstas.

Finalmente, la dimensión institucional describe la prueba del uso de la ciudad, en miras de la ciudad moderna globalizada. Aquello consignó en las mujeres entrando en la vejez un soporte que deja la experiencia comparada con la aventura de los viajes a otros países. Se comparan aquellas ciudades con la ciudad de residencia, permitiendo construir soportes citadinos en la vejez. Es decir, teniendo como parámetro la ciudad moderna e inclusiva de países desarrollados. Se consigna que lo anterior desarrolló soportes ante el bombardeo mediático que instaló el discurso de la seguridad ciudadana en los países de la región, lo que permite a mujeres de ciudades de Santiago y Montevideo hacer frente a la prueba del uso de la ciudad en la vejez.

Las mujeres en edades avanzadas identificaron como soporte el apoyo familiar para enfrentar la prueba, lo que les permitió, en particular, disponer de la compañía del marido o de las hijas especialmente cuando se ven impedidas de desenvolverse con autonomía en la ciudad. Se observó soportes institucionales para enfrentar la prueba de la ciudad, como la ley de discapacidad aplicable en ambos países, generando condiciones favorables para una ciudad más amigable. Se utilizan como estrategias para hacer frente a dicha prueba: el uso frecuente del metro en Santiago y uso de taxis en Montevideo.

Por último, se distinguen soportes legítimos en las mujeres para enfrentar las diversas pruebas expuestas en este estudio. Se constató, en la figura de la familia, un componente de época generacional de las mayores, desde donde la decisión o elección entre educación y matrimonio instituyó en ellas un soporte histórico generacional presente en su pasado. La experiencia del proceso de urbanización y la migración contribuyó a la formación de las grandes urbes regionales y la incorporación masiva de la mujer al mundo laboral, situando los relatos del pasado migrante entre los aportes generacionales.

\footnotetext{
${ }^{5}$ En relación a los mecanismos y estrategias de los soportes ilegítimos, es destacable mencionar cómo las mujeres buscaron en métodos alternativos de salud, que van desde la cultura homeopática hasta sistemas de creencias puritanos, diversas formas para enfrentar la prueba de la salud, diferenciándose sustancialmente según su condición, dueña de casa o jubilada y el nivel educativo. Precisamente, de la condición de cada cual va a depender como llegan a puerto las estrategias desarrolladas.
} 
El estudio reveló, además, estrategias que asumen y aplican las mujeres para intentar lidiar con la pobreza. Las mujeres hacen uso de mecanismos de transferencias de recursos económicos de subsidios dirigidos a familias con integrantes mayores. Se reconocieron, también, medidas laborales de capacitación, micro emprendimientos, acompañándose de estrategias sostenidas por una condición previsora propia de las mujeres en esta etapa de la vida. En cuanto a estrategias reconocidas socialmente, estas lograron consolidar un comportamiento individual entre las mujeres mayores, y también el reconocimiento socialmente requerido para sostenerlo en el tiempo.

\section{Conclusiones}

La investigación constató el desarrollo diferencial de los procesos históricos en las ciudades analizadas. Los desafíos comunes generacionales y las pruebas que ello conlleva, presentando soportes legítimos, haciendo de la experiencia generacional de las mujeres mayores en estudio una cartografía única del momento histórico en curso. Se identificaron modos actuales por los cuales los procesos de individuación se experimentan en las mujeres por medio de las pruebas, soportes y estrategias en la vejez.

Los soportes nos hablan de recursos que la propia generación posee al momento de hacer frente a las pruebas. La legitimidad de los soportes colocan los desafíos de la gran cantidad de pruebas enfrentadas por las mayores en la sociedad contemporánea en vías de su constitución como individuos, expuestas a niveles de fragilidad social y vulnerabilidad que acrecientan las complejidades coyunturales de la existencia en la etapa de la vejez. Lo expuesto estaría dando cuenta de una forma de vivir la vejez, con mecanismos que no entran en el mercado formal de valores de intercambio que la sociedad espera para esta etapa de la vida y menos para las mujeres mayores en ella. En este sentido, los procesos de individuación de las mujeres en contextos de envejecimiento poblacional y la fragilidad de los Estados proteccionistas de América del Sur, requieren de la profundización de estudios al respecto, que orienten las futuras políticas y matrices teóricas, que den cuenta de la compleja lectura de la vejez en ello.

En este escenario, se recomiendan líneas de estudios que indaguen con recursos y técnicas de historias de vida para profundizar en la interpretación y significado cultural que otorgan las mujeres sobre su propia vejez y el cotidiano reflejado en ello. El dinamismo y velocidad que adquiere en nuestras sociedades el fenómeno de la vejez, pareciera anunciar formas generacionales propiamente modernas que se debaten entre exigencias de individuación social por medio de diversos factores y contextos que llevan a una diferenciación en el proceso del envejecimiento de los individuos. Aquello, sin duda, invita a conocer y comparar 
el proceso de vejez desde otras vivencias como puede ser la de los hombres latinoamericanos. Se torna necesario ubicar el estudio de las políticas públicas en el centro del análisis de la vejez, dando un carácter estructural sin perder la configuración individual, la producción de individuo en sociedades envejecidas y la definición del espacio teórico en Latinoamérica de la individuación. Se propone potenciar futuros estudios por medio de temas tales como: el estudio de la vida cotidiana, por medio de historias de vida y otras técnicas, de la experiencia de la vejez moderna, la indagación de las estrategias y los mecanismos alternativos del conocimiento de las mujeres mayores, el conocimiento y sabiduría generacional que alimenta la cadena de saberes populares, que van perfeccionando la experiencia de la vejez, entre error y ensayo.

Cabe agregar que el estudio constató soportes generacionales de las mujeres mayores que circulan entre el espacio público y privado. Aquello refiere a un intercambio de códigos sociales construidos en la relación tradición/modernidad (en base a un pasado común), evidenciando una generación de mujeres que ven sus trayectorias interpeladas hoy, desde desafíos que dejan los grandes cambios de la sociedad salarial y los mandatos sociales que con ella forjaron sus individuos, tales como: la familia, el trabajo y la educación. Lo cual discute con la dinámica de la vida actual y su modo de ser moderno. En este escenario, la familia nuclear logra ser la tendencia que adquiere el nuevo espacio familiar donde envejece la población en ambas ciudades. Con familias monoparentales que comienzan a componer la imagen moderna donde habita la vejez, presenta un espacio aún reversible en la convivencia intergeneracional y el intercambio de imaginarios epocales del cuidado. De esta manera, en el recuerdo de la infancia o juventud que posee la sociedad en su conjunto de lo que fue o está siendo la transición de la pauta del cuidado de la familia extensiva, surge una alternativa de convivencia futura para sociedades envejecidas, que convoca al aprendizaje de prácticas de buen vivir y buen morir en las nuevas generaciones. 


\section{Referencias}

Achenbaum, A. (1997). Critical gerontology. En A. Jamieson, S. Harper, C. Victor (Eds.), Critical approaches to ageing and later life. Buckingham: Open University Press.

Araujo, K., y Martuccelli, D. (2012). Desafíos comunes. Retrato de la sociedad chilena y sus individuos. Santiago de Chile: LOM Ediciones.

Arber, S., y Ginn, J. (1990). The meaning of informal care: gender and the contribution of elderly people. Ageing and Sociery, 10(4), 429-454.

Arber, S., y Ginn, J. (1996). Relaciones de género y envejecimiento. Enfoque sociológico. Madrid: Narcea S.A. Ediciones.

Baltes, P. B. (1997). On the incomplete architecture of human ontogeny: Selection, optimization, and compensation as foundation of developmental theory. American Psychologist, 52, 366-380.

Barros, C. (1979). La vejez marginada, situación de los ancianos en Chile. Instituto de Sociología. Pontificia Universidad de Católica de Chile.

Bury, M. (1996). Envejecimiento, género y teoría sociológica. En S. Arber y J. Ginn (Coord.), Relación entre género y envejecimiento. Enfoque sociológico (pp. 35-54). Madrid: Nancea.

Butler, R.N. (1969). Age-Ism: another form of bigotry. The Gerontologist, 9, 243246.

Caradec, V. (2004). Les supports de I'ndividu vieillissant. Retour sur la notion de déprise. En V. Caradec y D. Martuccelli (Eds.), Matériaux pour une sociologie de l'individu. Perspectives et débats. Le regard sociologique. París: Presses Universitaires du Septentrion.

Castells, M. (2005). Globalización, desarrollo y democracia: Chile en el contexto mundial. Santiago: F.C.E.

CELADE/CEPAL. (2011). Mujeres mayores Indicadores para América Latina y el Caribe. Separata. Santiago de Chile. Cepal. Disponible en: http://www. gerontologia.org/portal/archivosUpload/Separata_Mujeres_E.pdf

CEPAL/ECLAC. (2014). Observatorio Demográfico, 2014.Comisión Económica para América Latina y el Caribe. Disponible en: https://repositorio.cepal.org/ bitstream/handle/11362/36854/1/S1420023_mu.pdf 
Debert, G. (1994). Gênero e envelhecimento. Revista de Estudos Feministas, 1, 33-51.

Elder, G., y Johnson, M. (2002). The life course and aging, challenges, lessons, and new directions. En R. A. Jr. Setterston (Ed.), Invitation to the life course: toward new understandings of later life, part 2 (pp. 49-81). Amityville, NY: Baywood.

Fernandez-Ballesteros, R. (2009). Envejecimiento activo. Contribuciones de la psicología. Madrid: Editorial Pirámide.

Ferrer, M. (2005). La población y el desarrollo desde un enfoque de derechos humanos: intersecciones, perspectivas y orientaciones para una agenda regional. Chile. Serie población y desarrollo N60. CEPAL/ CELADE- División de Población.

Freixas, A. (2007). Gerontología femenina: comprender la compleja y sutil vida de las mujeres mayores. Consultado en: https://www.gerontologia.org/portal/ information/showlnformation.php?idinfo=1006

Freixas, A., Luque, B., y Reina, A. (2009). El ciclo vital revisado. La vida de las mujeres mayores a la luz de los cambios sociales. Recerca, Revista de Pensament $i$ Anàlisi, 9, 59-80.

Giddens, A. (1994). Consecuencias de la modernidad. Madrid: Ed. Alianza Universitaria.

Herrero, F.G. (2001). Evolución de la institucionalización de los ancianos en España. Barcelona: Editorial GLOSA.

Huenchuan, S. (2012). Desarrollo institucional en materia de envejecimiento y vejez en la región. Seminario SENAMA/UCM. Conferencia. Santiago de Chile. Sin edición.

Lalive D'epinay, C. (1983). Vieillesses. Situations, itineraires et modes de vie des personnes âgées aujourd'hui. Saint-Saphori, Suisse: Editions Georgi.

Lalive D'epinay, C., Cavalli, S., y Guilley, E. (2005). Recorrido de vida y vejez: sobre la noción de fragilidad. Centro Inter-facultades de Gerontología. Ginebra: Universidad de Ginebra.

Levy, J. (1988). Intersections of gender and aging. The Sociological Quartely, 29(4), 479-486. 
Maqueira, V. (2002). Mujeres mayores en el siglo XXI. De la invisibilidad al protagonismo. Madrid: Ministerio de Trabajo y Asuntos Sociales, IMSERSO.

Martuccelli, D. (2010). La sociología en los tiempos del individuo. 1/2 vínculo. Entrevista a Danilo Martuccelli. 1(1). Disponible en: www.doblevinculo. wordpress.com

Martuccelli, D. (2007). Las tres vías del individuo sociológico. Cambio de Rumbo. Santiago de Chile: LOM Ediciones.

Martuccelli, D., y Singly, F. (2012). Las sociologías del Individuo. Serie individuos y ciencias sociales. Santiago de Chile: LOM Ediciones.

McMullin, J. (1996). Teoría de las relaciones de edad y género. En S. Arber, y J. Ginn, (Comp.), Relación entre género y envejecimiento. Enfoque sociológico. Madrid: Narcea.

MIDES. (2013). Plan nacional de envejecimiento y vejez 2013-2015. Gobierno del Uruguay. Montevideo. Uruguay. Instituto Nacional del Adulto Mayor.

Neugarten, B. (1999). Los significados de la edad. Barcelona: Herder.

ONU. (2012). Informe perspectiva de la población mundial. Naciones Unidas. Portada. Consultado el 25 de abril 2018 en: http://www.un.org/es/sections/ issues-depth/ageing/index.html

ONU. (2002). Plan mundial de envejecimiento. II Asamblea Mundial de Envejecimiento. Una sociedad para todas las edades. Santiago de Chile: CEPAL/CELADE.

Ortega, M. (2002). La vejez en la historia moderna. En V. Maqueira (Coord), Mujeres mayores en el siglo XXI: de la invisibilidad al protagonismo (pp. 5984). Madrid: IMSERSO.

Osorio, P. (2010). La persona mayor como sujeto de política. En S. Huenchuan (Ed.) Envejecimiento, género y políticas públicas: coloquio regional de expertos en Montevideo, Uruguay. Montevideo: Lucida Ediciones.

Peace, S., Holland, C., y Kellaher, L. (2006). Environment and identity in later life. Growing older. Maidenhead: Open University Press.

Pérez Díaz, J. (2000). La feminización de la vejez. RES, 104(3), 91-121.

Pérez Ortiz, L. (2003). Envejecer en femenino. Las mujeres mayores en España a comienzos del siglo XXI. Madrid: Instituto de la Mujer. 
Pérez Ortiz, L. (2006). Jubilación, género y envejecimiento. En J. Giró. (Coord.), Envejecimiento activo, envejecimiento en positivo (pp. 89-112). Logroño: Universidad de la Rioja, Colecciones Biblioteca de investigación.

Quadagno, J. (1999). Aging and the life course. An introduction to social gerontology. Florida: McGraw Hill.

Reinharz, S. (1989). Feminism and anti-ageism: emergent connection. En A. Herzog, K. Holden y M. Selzer (eds.), Health and economic status of older women. Nueva York: Baywood.

RLG.(2009). Haciaunasociedadparatodaslasedadesexperienciaslatinoamericanas sobre relaciones intergeneracionales. Selección de experiencias, concurso: una sociedad para todas las edades. Santiago de Chile: Red Latinoamericana de Gerontología.

Rodríguez, P. (2002). Mujeres mayores, género y protección social (o adónde conduce el amor. En V. Maqueira (Coord), Mujeres mayores en el siglo XXI: de la invisibilidad al protagonismo (pp. 87-136). Madrid: IMSERSO.

Yuni, J., y Urbano, C. (2008). Envejecimiento y género: perspectivas teóricas y aproximaciones al envejecimiento femenino. Revista Argentina de Sociología, 6(10), 151-169. 


\section{Como citar este artículo}

Obreque, R. (2018). Las pruebas de la vejez en mujeres. El caso de Santiago de Chile y Montevideo. Revista Pensamiento y Acción Interdisciplinaria, 4(1), 57-73. DOI: http://doi.org/10.29035/pai.4.1.57

\section{Dirección de correspondencia}

Regina Praxedes Obreque Morales

Socióloga.

Universidad de Santiago de Chile.

Contacto:

regina.obrequem@usach.cl

Recibido: 23/07/2018

Aceptado: 30/09/2018 